\title{
Pacific
}

Journal of

Mathematics

\section{ONE REMARK ON POLYNOMIALS IN TWO VARIABLES}

EnriQue Artal Bartolo and Pierrette Cassou-Noguès 


\title{
ONE REMARK ON POLYNOMIALS IN TWO VARIABLES
}

\author{
E. Artal Bartolo* and P. Cassou-Noguès
}

In this paper, we study some topological properties of rational polynomial maps $f: \mathbb{C}^{2} \rightarrow \mathbb{C}$. One can extend $f$ to a map $\varphi: \mathbf{X} \rightarrow \mathbb{P}^{1}$ where $\mathbf{X}$ is a smooth algebraic compactification of $\mathbb{C}^{2}$. The behaviour of $\varphi$ on the curve $\mathcal{D}:=\mathbf{X} \backslash \mathbb{C}^{2}$ contains all the information on the topology of $f$ at infinity. We study the relationship between the so-called horizontal componentes of $\mathcal{D}$, i.e., irreducible components $\mathrm{D}$ of $\mathcal{D}$ such that $\phi_{\mid} D$ is surjective.

In the article "Two remarks on polynomials in two variables" [5] S. Kaliman states two results on polynomial maps $f: \mathbb{C}^{2} \rightarrow \mathbb{C}$. The first one is a very nice result on the number of irreducible components of reducible fibers of a primitive polynomial (i.e., a polynomial such that its generic fiber is irreducible).

We introduce some notation in order to state the second result. Let $\mathbb{C}^{2} \subset$ $X$ a compactification of $\mathbb{C}^{2}$ where $X$ is a smooth rational compact surface and such that there exists a holomorphic map $\phi: X \rightarrow \mathbb{P}^{1}=\mathbb{C} \cup\{\infty\}$ which extends $f$.

Put $\mathcal{D}:=X \backslash \mathbb{C}^{2} ; \mathcal{D}$ is a curve whose irreducible components are smooth rational compact curves and all its singularities are ordinary double points. The dual graph of $\mathcal{D}$ is a tree.

Kaliman calls horizontal (resp. sections) an irreducible component $D \subset \mathcal{D}$ such that $\phi_{\mid D}$ is surjective (resp. bijective). We recall the second result of [5]:

If $f$ is primitive and the generic fiber of $f$ is a rational curve then there is at most one horizontal component which is not a section.

In this note we are going to give a counter-example to this result and we are going to point out where Kaliman's proof does not apply. In the last section we relate this problem to an article of P. Russell, [8].

\section{$\S 1$-A counter-example.}

Let us denote

$$
s(x, y)=1+x y, \quad p(x, y)=x s(x, y)+1, \quad u(x, y)=s(x, y)^{2}+y .
$$


We define:

$$
f(x, y):=p(x, y)^{4} u(x, y)^{2}-(p(x, y) s(x, y)+1)^{2} .
$$

For any $f$ there exists a canonical way to get $X$ and $\phi$. Let

$$
f(x, y)=a_{0}(x, y)+a_{1}(x, y)+\cdots+a_{d}(x, y)
$$

where $a_{j}(x, y)$ is a homogeneous form of degree $j, j=0,1, \ldots, d$, and $a_{d}(x, y) \neq 0$; let

$$
F(x, y, z):=\sum_{j=0}^{d} z^{d-j} a_{j}(x, y) .
$$

Consider the rational map

$$
\begin{aligned}
\phi_{0}: & \mathbb{P}^{2} \rightarrow \longrightarrow \quad \mathbb{P}^{1}=\mathbb{C} \cup\{\infty\} \\
{[x: y: z] } & \mapsto\left[F(x, y, z): z^{d}\right]=\frac{F(x, y, z)}{z^{d}},
\end{aligned}
$$

which is well-defined outside $A(f):=\left\{\left[x_{0}: y_{0}: 0\right] \mid a_{d}\left(x_{0}, y_{0}\right)=0\right\}$ (which is a finite set). This set is always contained in the line of infinity $L_{\infty}$ whose equation is $z=0$.

The composition $\pi: X \rightarrow \mathbb{P}^{2}$ of a suitable sequence of blowing-up maps (over $A(f)$ ) gives the required compactification where $\phi:=\phi_{0} \circ \pi$.

Let's compute $X$ and $\phi$ in our example; we have $d=20$ and $a_{20}(x, y)=$ $x^{12} y^{8}$ so $A(f)=\{[1: 0: 0],[0: 1: 0]\}$. Let us start with the blowing-up maps over $P:=[1: 0: 0]$; this is the origin of the chart defined by $x=1$. We write down $\phi_{0}$ in the coordinates $(y, z)$ of this chart:

$$
\phi_{0}(y, z)=\frac{F(1, y, z)}{z^{20}}=\frac{y^{8}+\cdots}{z^{20}},
$$

where "..." means terms of higher degree. We consider $\pi_{1}: Y_{1} \rightarrow \mathbb{P}^{2}$ the blowing-up of $P$.

Convention. We will identify a curve with its proper transform after a blowing-up, even in the notation, whenever it does not cause any confusion.

Let's call $A_{1}$ the exceptional locus of this blowing-up. It is easy to see from last formula that the only point of indeterminacy over $P$ of $\phi_{y 1}:=\phi_{0} \circ \pi_{1}$ is the infinitely near point $P_{1}$ of the line $\{y=0\}$. We choose a chart of $Y_{1}$ with coordinates $y_{1}, z_{1}$ such that $P_{1}$ is the origin of this chart and we can write down $\pi_{1}$ as follows:

$$
\pi_{1}\left(y_{1}, z_{1}\right)=\left(y_{1} z_{1}, z_{1}\right) .
$$


Note that $L_{\infty}$ does not intersect this chart and $A_{1}$ is defined by $z_{1}=0\left(P_{1}\right.$ is in $A_{1}$ but it is not in $L_{\infty}$ ). We write down $\phi_{y 1}$ in these coordinates:

$$
\phi_{y 1}\left(y_{1}, z_{1}\right)=\frac{F\left(1, y_{1} z_{1}, z_{1}\right)}{z_{1}^{20}}=\frac{f_{y 1}\left(y_{1}, z_{1}\right)}{z_{1}^{12}}=\frac{\left(y_{1}+z_{1}\right)^{8}+\cdots}{z_{1}^{12}} .
$$

We consider $\pi_{2}: Y_{2} \rightarrow Y_{1}$ the blowing-up of $P_{1}$. Let's call $A_{2}$ the exceptional locus of this blowing-up. It is easy to see that $A_{2}$ does not intersect $L_{\infty}$. From last formula the only point of indeterminacy over $P$ of $\phi_{y 2}:=\phi_{y 1} \circ \pi_{2}$ is the infinitely near point $P_{2}$ of the line $\left\{y_{1}+z_{1}=0\right\}$. We choose a chart of $Y_{2}$ with coordinates $y_{2}, z_{2}$ such that $P_{2}$ is the origin of this chart and we can write down $\pi_{2}$ as follows:

$$
\pi_{2}\left(y_{2}, z_{2}\right)=\left(\left(y_{2}-1\right) z_{2}, z_{2}\right)
$$

Note that $A_{2}$ is defined by $z_{2}=0$. We write down $\phi_{y 2}$ in these coordinates:

$$
\phi_{y 2}\left(y_{2}, z_{2}\right)=\frac{f_{y 1}\left(\left(y_{2}-1\right) z_{2}, z_{2}\right)}{z_{2}^{12}}=\frac{f_{y 2}^{\prime \prime \prime}}{\left.z_{2}^{4}, z_{2}\right)}=\frac{-z_{2}^{4}+\cdots}{z_{2}^{4}} .
$$

We consider $\pi_{3}: Y_{3} \rightarrow Y_{2}$ the blowing-up of $P_{2}$. Let $A_{3}$ be the exceptional locus of this blowing-up. It is easy to see that $A_{2}$ intersects neither $L_{\infty}$ nor $A_{1}$. From last formula the only point of indeterminacy over $P$ of $\phi_{y 3}:=$ $\phi_{y 2} \circ \pi_{3}$ is $P_{3}:=A_{2} \cap A_{3}$. We choose a chart of $Y_{3}$ with coordinates $y_{3}, z_{3}$ such that $P_{3}$ is the origin of this chart and we can write down $\pi_{3}$ as follows:

$$
\pi_{3}\left(y_{3}, z_{3}\right)=\left(y_{3}, y_{3} z_{3}\right)
$$

Note that $A_{3}$ is defined by $y_{3}=0$ while $A_{2}$ is $z_{3}=0$. We write down $\phi_{y 3}$ in these coordinates:

$$
\phi_{y 3}\left(y_{3}, z_{3}\right)=\frac{f_{y 2}\left(y_{3}, y_{3} z_{3}\right)}{y_{3}^{4} z_{3}^{4}}=\frac{f_{y 3}\left(y_{3}, z_{3}\right)}{z_{3}^{4}}=\frac{-z_{3}^{4}+\cdots}{z_{3}^{4}} .
$$

Note that $\phi_{y 3}\left(L_{\infty}\right)=\phi_{y 3}\left(A_{1}\right)=\phi_{y 3}\left(A_{2} \backslash\left\{P_{3}\right\}\right)=\infty=[1: 0]$, but we have $\phi_{y 3}\left(A_{3} \backslash\left\{P_{3}\right\}\right)=-1=[-1: 1]$.

If $\pi_{4}: Y_{4} \rightarrow Y_{3}$ is the blowing-up of $P_{3}$ then $\phi_{y 4}:=\phi_{y 3} \circ \pi_{4}$ has no point of indeterminacy in the exceptional locus $A_{4}$ of $\pi_{4}$; this curve separates $A_{2}$ and $A_{3}$; the restriction of $\phi_{y 4}$ is a surjective map of degree 4 (an horizontal component in the notation of S. Kaliman in [5]).

Let $Q$ be the transform of $[0: 1: 0]$ by the sequence $\pi_{4} \circ \pi_{3} \circ \pi_{2} \circ \circ \pi_{\Gamma}$. We consider also the transform of the chart $y=1$. In its coordinates $x, z$ we have:

$$
\phi_{y 4}(x, z)=\frac{F(x, 1, z)}{z^{20}}=\frac{x^{12}+\cdots}{z^{20}} .
$$


We consider $\sigma_{1}: X_{1} \rightarrow Y_{4}$ the blowing-up of $Q$. Let's call $B_{1}$ the exceptional locus of this blowing-up. It is easy to see that $B_{1}$ intersects $L_{\infty}$. From last formula the only point of indeterminacy of $\phi_{x 1}:=\phi_{y 4} \circ \sigma_{1}$ is the infinitely near point $Q_{1}$ of the line $x=0$ which does not lie in $L_{\infty}$. We choose a chart of $X_{1}$ with coordinates $x_{1}, z_{1}$ such that $Q_{1}$ is the origin of this chart and we can write down $\sigma_{1}$ as follows:

$$
\sigma_{1}\left(x_{1}, z_{1}\right)=\left(x_{1} z_{1}, z_{1}\right)
$$

Note that $B_{1}$ is defined by $z_{1}=0$. We write down $\phi_{x 1}$ in these coordinates:

$$
\phi_{x 1}\left(x_{1}, z_{1}\right)=\frac{F\left(x_{1} z_{1}, 1, z_{1}\right)}{z_{1}^{20}}=\frac{f_{x 1}\left(x_{1}, z_{1}\right)}{z_{1}^{8}}=\frac{z_{1}^{6}+\cdots}{z_{1}^{8}} .
$$

We consider $\sigma_{2}: X_{2} \rightarrow X_{1}$ the blowing-up of $Q_{1}$. Let's call $B_{2}$ the exceptional locus of this blowing-up. It is easy to see that $B_{2}$ intersects $B_{1}$ but it is disjoint with $L_{\infty}$. From last formula the only point of indeterminacy of $\phi_{x 2}:=\phi_{x 1} \circ \sigma_{2}$ is $Q_{2}:=B_{1} \cap B_{2}$. We choose a chart of $X_{2}$ with coordinates $x_{2}, z_{2}$ such that $Q_{2}$ is the origin of this chart and we can write down $\sigma_{2}$ as follows:

$$
\sigma_{2}\left(x_{2}, z_{2}\right)=\left(x_{2}, x_{2} z_{2}\right) \text {. }
$$

Note that $B_{2}$ is defined by $x_{2}=0$ and $B_{1}$ is $z_{2}=0$. We write down $\phi_{x 2}$ in these coordinates:

$$
\phi_{x 2}\left(x_{2}, z_{2}\right)=\frac{f_{x 1}\left(x_{2}, x_{2} z_{2}\right)}{x_{2}^{8} z_{2}^{8}}=\frac{f_{x 2}\left(x_{2}, z_{2}\right)}{x_{2}^{2} z_{2}^{8}}=\frac{\left(x_{2}+z_{2}\right)^{6}+\cdots}{x_{2}^{2} z_{2}^{8}} .
$$

We consider $\sigma_{3}: X_{3} \rightarrow X_{2}$ the blowing-up of $Q_{2}$. Let's call $B_{3}$ the exceptional locus of this blowing-up which separates $B_{1}$ and $B_{2}$. From last formula the only point of indeterminacy of $\phi_{x 3}:=\phi_{x 2} \circ \sigma_{3}$ is the infinitely near point $Q_{3}$ of the affine line $x_{2}=-z_{2}$. We choose a chart of $X_{3}$ with coordinates $x_{3}, z_{3}$ such that $Q_{3}$ is the origin of this chart and we can write down $\sigma_{3}$ as follows:

$$
\sigma_{3}\left(x_{3}, z_{3}\right)=\left(\left(x_{3}-1\right) z_{3}, z_{3}\right) .
$$

Note that $B_{3}$ is defined by $z_{3}=0$. We write down $\phi_{x 3}$ in these coordinates:

$$
\phi_{x 3}\left(x_{3}, z_{3}\right)=\frac{f_{x 2}\left(\left(x_{3}-1\right) x_{3}, z_{3}\right)}{\left(x_{3}-1\right)^{2} z_{3}^{10}}=\frac{f_{x 3}\left(x_{3}, z_{3}\right)}{\left(x_{3}-1\right)^{2} z_{3}^{4}}=\frac{-\left(x_{3} z_{3}\right)^{2}+\cdots}{\left(x_{3}-1\right)^{2} z_{3}^{4}} .
$$

We consider $\sigma_{4}: X_{4} \rightarrow X_{3}$ the blowing-up of $Q_{3}$. Let's call $B_{4}$ the exceptional locus of this blowing-up which intersects only $B_{3}$. It is easy to see that $B_{4}$ is an horizontal component such that the restriction of $\phi_{x 4}:=\phi_{x 3} \circ \sigma_{4}$ to $B_{4}$ is a map of degree 2 ; we can also check that $Q_{5}:=B_{4} \cap B_{3}$ is a point 
of indeterminacy of $\phi_{x 4}$. We choose a chart of $X_{4}$ with coordinates $x_{4}, z_{4}$ such that $Q_{4}$ is the origin of this chart and we can write down $\sigma_{4}$ as follows:

$$
\sigma_{4}\left(x_{4}, z_{4}\right)=\left(x_{4}, x_{4} z_{4}\right) .
$$

Note that $B_{4}$ is defined by $x_{4}=0$ and $B_{3}$ is $z_{4}=0$. We write down $\phi_{x 4}$ in these coordinates:

$$
\phi_{x 4}\left(x_{4}, z_{4}\right)=\frac{f_{x 3}\left(x_{4}, x_{4} z_{4}\right)}{\left(x_{4}-1\right)^{2} x_{4}^{4} z_{4}^{4}}=\frac{f_{x 4}\left(x_{4}, z_{4}\right)}{\left(x_{4}-1\right)^{2} z_{4}^{4}}=\frac{\left(x_{4}-z_{4}\right)\left(x_{4}+z_{4}\right)+\cdots}{\left(x_{4}-1\right)^{2} z_{4}^{4}} .
$$

We consider $\sigma_{5}: X_{5} \rightarrow X_{4}$ the blowing-up of $Q_{4}$. Let's call $B_{5}$ the exceptional locus of this blowing-up which separates $B_{3}$ and $B_{4}$. It is easy to see that there are two points of indeterminacy of $\phi_{x 5}:=\phi_{x 4} \circ \sigma_{5}$; let's denote $Q_{5}$ the infinitely near point of the affine line $x_{4}=z_{4}$ and $Q_{5}^{\prime}$ the one of $x_{4}=-z_{4}$. These points are the two points of indeterminacy. They behave in the same way so we study only $Q_{5}$. We choose a chart of $X_{5}$ with coordinates $x_{5}, z_{5}$ such that $Q_{5}$ is the origin of this chart and we can write down $\sigma_{5}$ as follows:

$$
\sigma_{5}\left(x_{5}, z_{5}\right)=\left(x_{5}, x_{5}\left(z_{5}+1\right)\right) .
$$

Note that $B_{5}$ is defined by $x_{5}=0$. We write down $\phi_{x 5}$ in these coordinates:

$$
\begin{aligned}
\phi_{x 5}\left(x_{5}, z_{5}\right) & =\frac{f_{x 4}\left(x_{5}, x_{5}\left(z_{5}+1\right)\right.}{\left(x_{5}-1\right)^{2} x_{5}^{4}\left(z_{5}+1\right)^{4}} \\
& =\frac{f_{x 5}\left(x_{5}, z_{5}\right)}{\left(x_{5}-1\right)^{2}\left(z_{5}+1\right)^{4} x_{5}^{2}} \\
& =\frac{-2\left(3 x_{5}+z_{5}\right)+\cdots}{\left(x_{5}-1\right)^{2}\left(z_{5}+1\right)^{4} x_{5}^{2}} .
\end{aligned}
$$

It is easy to see that if $\sigma_{6}: X_{6} \rightarrow X_{5}$ is the blowing-up of $Q_{5}$ then $\phi_{x 6}:=\phi_{x 5} \circ \sigma_{6}$ has only one point of indeterminacy $Q_{6}$ in the exceptional locus $B_{6}$ of $\sigma_{6}$. It is not difficult to show that if $\sigma_{7}: X_{7} \rightarrow X_{6}$ is the blowing-up of $Q_{6}$ then its exceptional locus $B_{7}$ is a section of $\phi_{x 7}:=\phi_{x 6} \circ \sigma_{7}$.

Let's denote $\sigma_{6}^{\prime}: X_{6}^{\prime} \rightarrow X_{7}$ and $\sigma_{7}^{\prime}: X_{7}^{\prime} \rightarrow X_{6}^{\prime}$ the corresponding blowingup maps over $Q_{5}^{\prime}$ where $\phi_{x 6}^{\prime}:=\phi_{x 7} \circ \sigma_{6}^{\prime} \phi_{x 7}^{\prime}:=\phi_{x 6}^{\prime} \circ \sigma_{7}^{\prime}$. Then if we denote

$$
\pi:=\sigma_{7}^{\prime} \circ \sigma_{6}^{\prime} \circ \sigma_{7} \circ \cdots \circ \sigma_{1} \pi_{4} \circ \cdots \circ \pi_{1}, \quad X:=X_{7}^{\prime}, \quad \phi:=\phi_{x 7}^{\prime} .
$$

Then we have found the required compactification of $\mathbb{C}^{2}$.

Let us summarize the results above. We draw in Figure 1 the dual graph of $\mathcal{D}=X \backslash \mathbb{C}^{2}$; note that $\mathcal{D}$ is the preimage by $\pi$ of $L_{\infty} \subset \mathbb{P}^{2}$. Weights are self-intersections in $X$; we recall that the exceptional locus of a blowing-up 
has self-intersection -1 and if a smooth curve passing through the center of the blowing-up has self-intersection $e$, then its proper transform has selfintersection $e-1$.

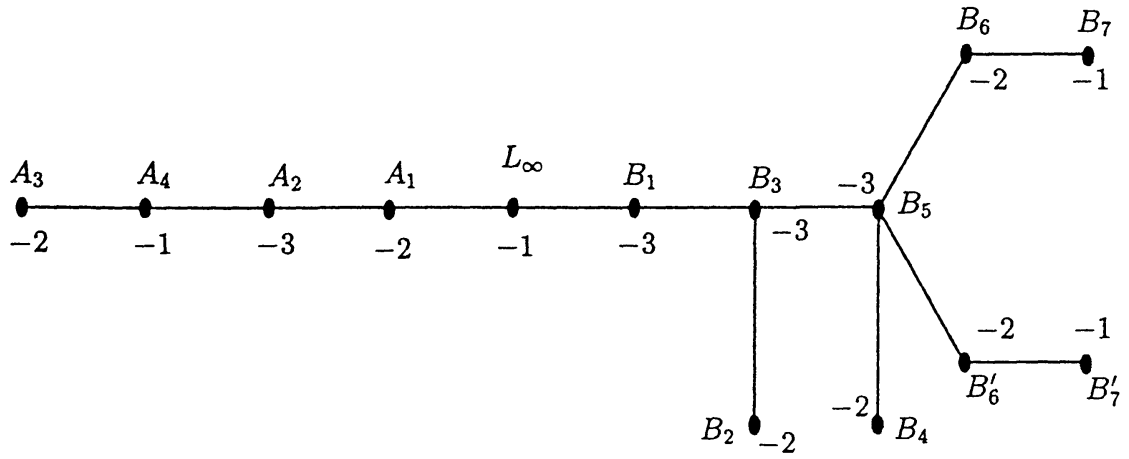

Figure 1.

We are following the next conventions:

- $\pi\left(A_{j}\right)=[1: 0: 0]$;

- $\pi\left(B_{j}\right)=\pi\left(B_{k}^{\prime}\right)=[0: 1: 0], j=1, \ldots, 7, k=6,7$;

- $\phi\left(L_{\infty}\right)=\phi\left(A_{1}\right)=\phi\left(A_{2}\right)=\phi\left(B_{j}\right)=\phi\left(B_{6}^{\prime}\right)=\infty=[1: 0], j=1, \ldots, 6$;

- $\phi\left(A_{3}\right)=-1=[-1: 1]$

- $B_{7}$ and $B_{7}^{\prime}$ are sections;

- $A_{4}$ is an horizontal component of degree 4 and

- $B_{4}$ is an horizontal component of degree 2 .

Proposition. $f$ is a primitive polynomial.

Proof. If $f$ would be not primitive then there exists $p \in \mathbb{C}[t]$ and $g \in \mathbb{C}[x, y]$ such that $f(x, y)=p(g(x, y))$ and $\operatorname{deg} p>1$. This fact uses Stein factorisation ([1, pp. 25-26]). An incomplete proof may be found in [4]. It is easy to see that if we construct $\psi_{0}: \mathbb{P}^{2} \rightarrow \mathbb{P}^{1}$ for $g$ in the same way as we construct $\phi$ for $f$ then the map $\psi:=\psi_{0} \circ \pi: X \rightarrow \mathbb{P}^{1}$ is holomorphic and it is an extension of $g$.

We deduce that the horizontal components are the same in the two cases: in fact if $D \subset \mathcal{D}$ is an irreducible component we have $\phi_{\mid D}=p \circ \psi_{\mid D}$, where $p$ is considered as a map $\mathbb{P}^{1} \rightarrow \mathbb{P}^{1}$ such that $p(\infty)=\infty$.

Then for an horizontal component $D$ we have that $\operatorname{deg} \phi_{\mid D}=\operatorname{deg} p \operatorname{deg} \psi_{\mid D}$. The existence of sections implies that $\operatorname{deg} p=1$ so $f$ is primitive. 
Proposition. The generic fiber of $f$ is rational.

Proof. Note that the general fiber is smooth; if we consider its compactification in $\mathbb{P}^{2}$ its singularities are on $A(f)$. It is known (see [2, pp. 569 and 574] and $[6$, p. 85]) that the genus of an irreducible plane projective $C$ curve is $(d-1)(d-2) / 2-\sum_{P} \delta_{P}$, where $d$ is the degree, the sum is over the singular points and $\delta_{P}$ may be calculated as follows.

We recall that the sequence of multiplicities of a singularity is the list of the multiplicities of the proper transform of the singularity at all infinitely near points (see [2, p. 504]). Then

$$
\delta_{P}=\sum_{P_{i}} \frac{m\left(P_{i}\right)\left(m\left(P_{i}\right)-1\right)}{2}
$$

where the sum is over the infinitely near points of $C$ at $P$ and $m\left(P_{i}\right)$ is their multiplicity.

Note that we have constructed a resolution of the singular points of the compactification of the general fiber as its proper transform in $X$ is smooth. We can get the sequence of multiplicities from this construction. In $[1: 0$ : $0]$ the generic fiber has four branches and the sequence of multiplicities is $8,8,4,4,\{1,1,1,1\} ;$ in $[0: 1: 0]$ the generic fiber has also four branches and the sequence of multiplicities is $12,6,6,4,\{1,1,2\},\{1,1,1,1\}$.

Putting these multiplicities in the formula we get zero.

Corollary. $f$ is a counterexample to Kaliman's second result in [5].

Finally we shoud note that the number of reducible fibers of $f$ is equal to three (each of them with exactly two components); it agrees with the first result of Kaliman in [5]. Using that $p s=x u+1$ we have

$$
\begin{aligned}
f & =(p u-p s-1)(p u+p s+1), \\
f+1 & =p\left(p^{3} u^{2}-s(p s+2)\right) \\
f+4 & =u\left(p^{3} u-x(x u+4)\right)
\end{aligned}
$$

\section{$\S 2 .-$ Eisenbud-Neumann diagrams and horizontal sections.}

These computations may be done using Eisenbud-Neumann diagrams of the link at infinity of the generic fiber of $f$. In order to find the diagram in Figure 2 we may use the computations for the compactification of $\mathbb{C}^{2}$ to find the Puiseux expansions of the singularities of the generic fiber of $f$ at 
infinity. Using [3, pp. 50] and [7, p. 450] we get the diagram in Figure 2, called $D_{E N}$.

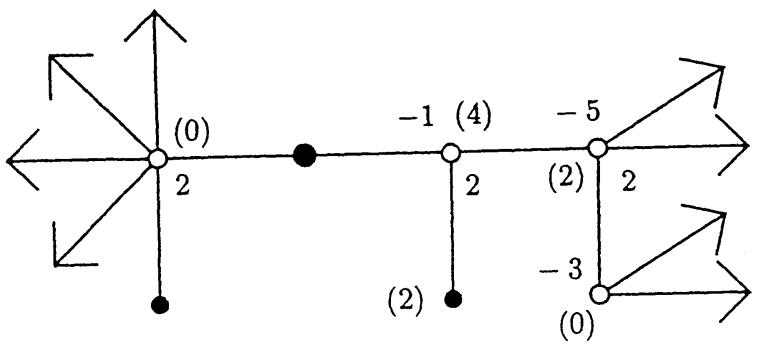

\section{Figure 2.}

We can see from $D_{E N}$ that $f$ is primitive. We must construct the fibered multilink associated to $f$, see [7], and verify that its fiber is connected using $[3$, p. 37]. The generic fiber of $f$ is an $n$-punctured Riemann surface since arrows in the EN-diagram correspond to punctures. The EN-diagram (Theorem 4.3 from [7]) also enables us to compute the Euler characteristic of the generic fiber which is -6 . Hence the genus of the generic fiber is 0 .

Remark. In our example horizontal components which are not sections are detected at vertices $v$ in $D_{E N}$ such that $l_{v}=0$ (weight in brackets). In the general case we detect in this way all horizontal components where $f$ is not equisingular; an horizontal component $D$ is equisingular if for all $t \in \mathbb{C}$ the closure of $f^{-1}(t)$ in $X$ intersects $D$ at exactly one point and transversally. We are going to explain how.

Let us consider the diagram in Figure 1. If we attach four arrows to the vertex $A_{4}$, two arrows to $B_{4}$ and one arrow to $B_{7}$ and $B_{7}^{\prime}$ then we obtain a plumbing diagram of the link at infinity of $f$. The dictionnary between these two kinds of diagrams may be found in [3, Chap. 5].

This diagram can be interpretted as a resolution diagram of the singularities of the closure of the generic fiber in $\mathbb{P}^{2}$; this diagram includes also the proper transform of the line at infinity. In this way is not minimal: we may eliminate vertices with weight -1 and belonging to at most two edges (this operation increases by one the weight of their neighbours). We call $D_{p b}$ the diagram obtained in this way. This construction correspond to a sequence of contractions of $(-1)$-rational curves which we denote $\tilde{\pi}: X \rightarrow \widetilde{X} ; D_{p b}$ is the dual graph of the curve $\widetilde{\mathcal{D}}=\tilde{\pi}(\mathcal{D})$. Let's denote $\tilde{\phi}: \tilde{X} \rightarrow \mathbb{P}^{1}$ the meromorphic function such that $\phi=\tilde{\phi} \circ \tilde{\pi}$. Let us emphasize that $\hat{\pi}: \tilde{X} \rightarrow \mathbb{P}^{2}$ is the minimal embedded resolution of the singularities of the closure of the 
generic fiber in $\mathbb{P}^{2}$ where $\hat{\pi}$ is the compositon of the blowing-up maps such that $\pi=\hat{\pi} \circ \tilde{\pi}$. Let us note that in general $\tilde{\phi}$ is not everywhere defined.

Lemma. Let $D_{E N}$ be the Eisenbud diagram of the link at infinity of the generic fiber of $f$. Let $D_{p b}$ be the dual tree of the total transform of the line at infinity in the minimal embedded resolution of the singularities of the closure of the generic fiber in $\mathbb{P}^{2}$.

Then there exists a natural bijection between vertices with attached arrows in $D_{E N}$ and $D_{p b}$ such that if $v$ is such a vertex in $D_{E N}$ and $D_{v}$ is the corresponding vertex in $D_{p b}$ then $l_{v}$ is the multiplicity of $D_{v}$ as pole of the meromorphic function $\tilde{\phi}$.

Proof. We recall the definition of $l_{v}$. Each vertex of $D_{E N}$ corresponds to a submanifold of $S^{3}$ which is an oriented fibration in circles. Let's denote $f_{v}$ a fiber corresponding to $v$ and disjoint from $K_{f}$ which is the link at infinity of a generic fiber of $f$. Then $l_{v}$ is the linking number of $f_{v}$ and $K_{f}$. Let us take an oriented Seifert surface $F_{f}$ of $K_{f}$ which is transversal to $f_{v}$; by definition of linking number $l_{v}$ is the algebraic intersection number of $f_{v}$ and $F_{f}$.

We are going to take a model of $S^{3}$ in $\mathbb{C}^{2}$ adapted to our situation. Note that $\widetilde{X} \backslash \widetilde{\mathcal{D}}=\mathbb{C}^{2}$ and the boundary of a well-chosen tubular neighbourhood of $\widetilde{\mathcal{D}}$ is $S^{3}$. Let's call $\mathcal{D}_{\infty}$ the union of irreducible components of $\widetilde{\mathcal{D}}$ whose image by $\tilde{\phi}$ is $\infty$. We take $\tilde{\phi}^{-1}\left(D_{R}\right)$ as tubular neighbourhood of $\mathcal{D}_{\infty}$ where

$$
D_{R}:=\{t \in \mathbb{C}|| t \mid \geq R\} \cup\{\infty\} \subset \mathbb{P}^{1},
$$

and $R$ is big enough.

We may choose a Hermitian metric in $\tilde{X}$ such that:

(a) For each double point of $\widetilde{\mathcal{D}}$ there exists local coordinates $(u, v)$ in a neignbourhood $U$ of this point such that $\widetilde{\mathcal{D}} \cup U$ has $u v=0$ as equation and the restriction of the metric to $U$ is the standard one with respect to $(u, v)$.

(b) If the double point is in one component $D_{1} \subset \widetilde{\mathcal{D}}$ whose equation is $u=0$ and another component $D_{2} \notin \widetilde{\mathcal{D}}$ then $\phi_{\mid U}=u^{-1}$.

For each component of $\widetilde{\mathcal{D}}$ which is not in $\mathcal{D}_{\infty}$ we choose small tubular neighbourhoods in this metric. The union of these tubular neighbourhoods with $\tilde{\phi}^{-1}\left(D_{R}\right)$ produces a tubular neighbourhood of $\widetilde{\mathcal{D}}$. Let's take $S^{3}$ as the boundary of this big tubular neighbourhood.

Let's take as generic fiber $F_{f}$ the intersection of $f^{-1}(R)$ with $S^{3}$. Let us. take a vertex $v$ such that $D_{v}$ is not sent to $\infty$; then we may choose $f_{v}$ disjoint from $F_{f}$ and so $l_{v}=0\left(f_{v}\right.$ is the boundary of a meridian disk of $\left.D_{v}\right)$. In this case as the generic point of $D_{v}$ is not a pole its multiplicity as pole is also zero. 
If $D_{v} \subset \mathcal{D}_{\infty}$ then choose a point $P \in D_{v}$ which is smooth in $\widetilde{\mathcal{D}} \cup \overline{f^{-1}(t)}$, for a generic $t \in \mathbb{C}$. We can choose analytic coordinates $u, v$ around $P$ such that the equation of $D_{v}$ is $v=0$ and $\tilde{\phi}(u, v)=v^{-a}$ where $a$ is the multiplicity of $D_{v}$ as pole of $\tilde{\phi}$. Note that we may take $f_{v}=\left\{(u, v)|u=0| v \mid,=R^{-a}\right\}$. Then the algebraic intersection of $F_{f}$ and $f_{v}$ is equal to $a$.

Let us use this lemma to prove the remark. We recall that $l_{v} \geq 0$ for regular links. If $l_{v}>0$ we deduce from the lemma that the generic point of $D_{v}$ is sent to infinity by $\tilde{\phi}$. A generic fiber of $\tilde{\phi}$ is smooth at $D_{v}$ and transversal to it; let's denote $P_{1}, \ldots, P_{r}$ the points of this generic fiber in $D_{v}$. These points are points of indeterminacy of $\tilde{\phi}$ as two different fibers pass through them (a generic one and $\tilde{\phi}^{-1}(\infty)$ ). As $\hat{\pi}$ is an embedded resolution of the closure of the generic fiber in $\mathbb{P}^{2} P_{i}$ is a smooth point of $\tilde{\phi}^{-1}(t), t \in \mathbb{C}$, and each fiber is transversal to $D_{v}$. The intersection number of two fibers at $P_{i}$ is equal to $l_{v}$. Then blowing up over each $P_{i} l_{v}$ times, we obtain $r$ equisingular horizontal components which are sections.

If $l_{v}=0$ there are two possibilities for $D_{v}$. It may be an horizontal component; minimality of $D_{p b}$ and the fact that this graph is a tree assure that this component is not equisingular and its degree equals the number of attached arrows. It may be also a component on which $\phi$ is constant and finite. In this case we cannot have arrows of the generic fiber attached to this component because only one fiber can intersect this component.

\section{§3.-About Kaliman's proof.}

The scheme of Kaliman's proof of this second result is as follows (see [5]): Lemma $4 \Rightarrow$ Lemma 5 and then the result follows from Lemma 5 and Corollary 3. Let us recall the statement of Lemma 4 :

Lemma 4 [5]. Let $f$ be a polynomial whose generic fiber is rational and let $\phi: X \rightarrow \mathbb{P}^{1}$ an extension of $f$. Let $H_{1}, H_{2}$ two horizontal components of $\mathcal{D}$. Then there is a commutative diagram

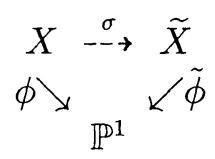

such that

(1) $\tilde{\phi}: \tilde{X} \rightarrow \mathbb{P}^{1}$ is an extension of $f$ where $\tilde{X}$ is a rational smooth compact surface and $\sigma$ is a birational isomorphism.

(2) The restriction of $\sigma$ to $\phi^{-1}(\mathbb{C})$ is a regular isomorphism from $\phi^{-1}(\mathbb{C})$ to $\tilde{\phi}^{-1}(\mathbb{C})$.

(3) $\tilde{\phi}^{-1}(\infty)$ is irreducible. 
(4) If $\widetilde{H}_{k}$ is the closure of the transform of $H_{k}$ by $\sigma, k=1,2$, then $\widetilde{H}_{1} \cap$ $\widetilde{H}_{2} \cap \tilde{\phi}^{-1}(\infty)=\emptyset$.

As Kaliman says it is easy to see that (applying Kodaira-Morrow's theorem) there exists a commutative diagram

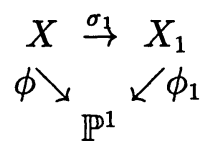

satisfying (1),(2) and (3) ( $\sigma_{1}$ is regular). In our case $\sigma_{1}$ is obtained collapsing one after the other the curves $L_{\infty}, A_{1}, B_{1}, A_{2}, B_{3}, B_{2}, B_{5}, B_{6}$.

In order to simplify the notation we keep the notation from Figure 1 for the proper transforms of the curves in $X$. We have:

- $B_{6}^{\prime}=p_{1}^{-1}(\infty)$,

- $P=B_{6}^{\prime} \cap A_{4} \cap B_{4}$,

- $\left(A_{4}, P\right)$ is a simple singularity of type $\mathbb{A}_{4}$,

- $\left(B_{4}, P\right)$ is smooth,

- $\left(B_{4} \cdot A_{4}\right)_{P}=5$ and

- $\left(B_{4} \cdot B_{6}^{\prime}\right)_{P}=2$.

Let us take $H_{1}=A_{4}$ and $H_{2}=B_{4}$. Kaliman asserts that if $H_{1} \cap H_{2} \cap$ $\phi_{1}^{-1}(\infty) \neq \emptyset$ then we may blow up $P$ and then contract the proper transform of $B_{6}^{\prime}$ (a-1-curve). We obtain $\phi_{2}: X_{2} \rightarrow \mathbb{P}^{1}$ and $\sigma_{2}$ satisfying (1), (2) and (3); he claims that the intersection number of the proper transforms of $H_{1}$ and $\mathrm{H}_{2}$ decrease and we can apply induction.

We see in our case that the intersection number does not decrease (it does for the blowing-up but as the curves are tangents to $B_{6}^{\prime}$ it increases with the contraction). In any case it is not possible to separate $H_{1}$ and $H_{2}$ with the hypothesis of Lemma 4 because if it would be true we may apply the scheme of Kaliman's proof showing that $f$ has at most one horizontal component which is not a section and this statement is not true.

\section{§4.- Remarks on an article of Russell.}

In [8] the author constructs an example of bad field generator, i.e., a polynomial $f \in \mathbb{C}[x, y]$, such that its generic fiber is rational and such that there is no polynomial $g \in \mathbb{C}[x, y]$ verifying $k(f, g)=k(x, y)$. This property is related with the non-existence of sections: a rational polynomial is a bad field generator if and only if no horizontal component is a section. The example given by Russell is a polynomial of degree 21 ; it has only two horizontal 
components, one of degree 3 and the other one of degree 2. It gives also a counter-example to Kaliman's theorem. In fact, from the above characterisation of bad field generator, [8,2.2], we deduce that all bad field generators are counter-examples to Kaliman's theorem; note that our counter-example is a good field generator because there are two sections. We write down his polynomial because the polynomial which appears in $[\mathbf{8}, \mathrm{p} .324]$ is not the right one:

$$
\begin{aligned}
f(x, y):= & y^{3}(x y+1)^{9}+4 x^{7} y^{9}+25 x^{6} y^{8}+66 x^{5} y^{7} \\
& +6 x^{5} y^{6}+95 x^{4} y^{6}+23 x^{4} y^{5}+80 x^{3} y^{5}+34 x^{3} y^{4}+4 x^{3} y^{3} \\
& +39 x^{2} y^{4}+24 x^{2} y^{3^{*}}+7 x^{2} y^{2}+10 x y^{3}+8 x y^{2^{*}}+3 x y+x+y^{2}+y^{*}
\end{aligned}
$$

(The ${ }^{*}$ shows the coefficients which do not match with [8].) From the first remark of [5] we deduce that this polynomial has one factorisation. In fact we have:

$$
\begin{aligned}
f:=\left(y^{2}(x y+1)^{4}+y(2 x y+1)(x y+1)+1\right) \\
\\
\times\left(y(x y+1)^{5}+2 x y(x y+1)^{2}+x\right) .
\end{aligned}
$$

\section{References}

[1] W. Barth, E. Peters and A. Van de Ven, Compact Complex Surfaces, Ergebnisse der Mathematik und ihre Grenzgebiete 3, Folge-Band 4, Springer Verlag, BerlinHeildelberg, (1984).

[2] E. Brieskorn and H. Knörrer, Plane Algebraic Curves, Birkhäuser Verlag, Basel, (1985).

[3] D. Eisenbud and W.D. Neumann, Three-Dimensional link theory and invariants of plane curve singularities, Annals of Mathematic Studies 101, Princeton Univ. Press, Princeton N.Y., (1987).

[4] M. Furushima, Finite groups of polynomial automorphisms in the complex affine plane (I), Memoirs of the Fac. of Sci. Kyushu Univ. Ser. A, 36 (1982), 85-105.

[5] S. Kaliman, Two remarks on polynomial in two variables, Pacific J. Math., 154 (1992), 285-295.

[6] J. Milnor, Singular Points of Complex Hypersurfaces, Annals of Mathematic Studies 61, Princeton Univ. Press, Princeton N.Y., (1968).

[7] W.D. Neumann, Complex algebraic plane curves via their links at infinity, Invent. Math., 98 (1989), 445-489.

[8] P. Russell, Good and bad field generators, J. Math. Kyoto Univ., 17 (1977), 319-331. 
Received May 20, 1994. The first author was partially supported by CAICYT PB91-0370C02-01.

UNIVERSIDAD DE ZARAGOZA

E-50009 Zaragoza, Spain

E-mail address: artal@posta.unizar.es

AND

UNIVERSITÉ DE BoRdEAUX I

351 COURS DE LA LIBÉRATION

F-33405 Talence, France

E-mail address: cassou@math.u-bordeaux.fr 



\title{
PACIFIC JOURNAL OF MATHEMATICS
}

\author{
Founded in 1951 by
}

\author{
$\begin{array}{ll}\text { E. F. Beckenbach (1906-1982) } & \text { F. Wolf (1904-1989) }\end{array}$
}

\section{EDITORS}

Sun-Yung A. Chang (Managing Editor) Robert Finn University of California

Los Angeles, CA 90095-1555

pacific@math.ucla.edu

\section{F. Michael Christ}

University of California

Los Angeles, CA 90095-1555

christ@math.ucla.edu

Nicholas Ercolani

University of Arizona

Tucson, AZ 85721

ercolani@math.arizona.edu
Stanford University

Stanford, CA 94305

finn@gauss.stanford.edu

Steven Kerckhoff

Stanford University

Stanford, CA 94305

spk@gauss.stanford.edu

Martin Scharlemann

University of California

Santa Barbara, CA 93106

mgscharl@math.ucsb.edu

\section{Gang Tian}

Massachusettes Institute of Technology

Cambridge, MA 02139

tian@math.mit.edu

\section{S. Varadarajan University of California Los Angeles, CA 90095-1555 vsv@math.ucla.edu \\ Dan Voiculescu \\ University of California \\ Berkeley, CA 94720 \\ dvv@math.berkeley.edu}

\section{SUPPORTING INSTITUTIONS}

ACADEMIA SINICA, TAIPEI

CALIF. INST. OF TECHNOLOGY

CHINESE UNIV. OF HONG KONG

HONG KONG UNIV. OF SCI. \& TECH.

KEIO UNIVERSITY

MACQUARIE UNIVERSITY

MATH. SCI. RESEARCH INSTITUTE

NEW MEXICO STATE UNIV.

OREGON STATE UNIV.

PEKING UNIVERSITY

RITSUMEIKAN UNIVERSITY

STANFORD UNIVERSITY

\author{
TOKYO INSTITUTE OF TECHNOLOGY \\ UNIVERSIDAD DE LOS ANDES \\ UNIV. OF ARIZONA \\ UNIV. OF BRITISH COLUMBIA \\ UNIV. OF CALIF., BERKELEY \\ UNIV. OF CALIF., DAVIS \\ UNIV. OF CALIF., IRVINE \\ UNIV. OF CALIF., LOS ANGELES \\ UNIV. OF CALIF., RIVERSIDE \\ UNIV. OF CALIF., SAN DIEGO \\ UNIV. OF CALIF., SANTA BARBARA
}

UNIV. OF CALIF., SANTA CRUZ

UNIV. OF HAWAII

UNIV. OF MELBOURNE

UNIV. OF MONTANA

UNIV. NACIONAL AUTONOMA DE MEXICO

UNIV. OF NEVADA, RENO

UNIV. OF OREGON

UNIV. OF SOUTHERN CALIFORNIA

UNIV. OF UTAH

UNIV. OF WASHINGTON

WASHINGTON STATE UNIVERSITY

The supporting Institutions listed above contribute to the cost of publication of this Journal, but they are not owners or publishers and have no responsibility for its contents or policies.

Manuscripts must be prepared in accordance with the instructions provided on the inside back cover.

The table of contents and the abstracts of the papers in the current issue, as well as other information about the Pacific Journal of Mathematics, may be found on the Internet at http://www.math.uci.edu/pjm.html.

The Pacific Journal of Mathematics (ISSN 0030-8730) is published monthly except for July and August. Regular subscription rate: $\$ 245.00$ a year (10 issues). Special rate: $\$ 123.00$ a year to individual members of supporting institutions.

Subscriptions, back issues published within the last three years and changes of subscribers address should be sent to Pacific Journal of Mathematics, P.O. Box 4163, Berkeley, CA 94704-0163, U.S.A. Prior back issues are obtainable from Kraus Periodicals Co., Route 100, Millwood, NY 10546.

The Pacific Journal of Mathematics at the University of California, c/o Department of Mathematics, 981 Evans Hall, Berkeley, CA 94720 (ISSN 0030-8730) is published monthly except for July and August. Second-class postage paid at Berkeley, CA 94704, and additional mailing offices. POSTMASTER: send address changes to Pacific Journal of Mathematics, P.O. Box 6143, Berkeley, CA 94704-0163.

\section{PUBLISHED BY PACIFIC JOURNAL OF MATHEMATICS at University of California,} Berkeley, CA 94720, A NON-PROFIT CORPORATION

This publication was typeset using AMS-LATEX,

the American Mathematical Society's TEX macro system.

Copyright (C) 1995 by Pacific Journal of Mathematics 


\section{PACIFIC JOURNAL OF MATHEMATICS}

\section{Volume $176 \quad$ No. $2 \quad$ December 1996}

One remark on polynomials in two variables

ENRIQUe ARTAl BARTOLO and PIERRETTE CASSOU-NOGUÈS

Divergence of the normalization for real Lagrangian surfaces near complex tangents XIANGHONG GONG

Classification of the stable homotopy types of stunted lens spaces for an odd prime JESUS GONZALEZ

Plancherel formulae for non-symmetric polar homogeneous spaces

JING-SONG HUANG

A uniqueness theorem for the minimal surface equation

JENN-FANG HWANG

Differential Galois groups of confluent generalized hypergeometric equations: an approach

using Stokes multipliers

Claudine Mitschi

Oscillatory theorem and pendent liquid drops

KIMIAKI NARUKAWA and TAKASHI SUZUKI

Local and global plurisubharmonic defining functions

ALAN NOELL

Specializations and a local homeomorphism theorem for real Riemann surfaces of rings

M. J. DE LA PUENTE

Eigenvalue comparisons in graph theory

GREGORY T. QUENELL

Applications of loop groups and standard modules to Jacobians and theta functions of isospectral curves

WILLI SCHWARZ

Bridged extremal distance and maximal capacity

ROBERT E. THURMAN

Imbedding and multiplier theorems for discrete Littlewood-Paley spaces

IGOR E. VERBITSKY

On constrained extrema

THOMAS VOGEL

Heat flow of equivariant harmonic maps from $\mathbb{B}^{3}$ into $\mathbb{C P} \mathbb{P}^{2}$

YUANLONG XIN

Proof of Longuerre's theorem and its extensions by the method of polar coordinates

ZHIHONG YU

Correction to: "Special generating sets of purely inseparable extension fields of unbounded exponent"

BONIFACE IHEMOTUONYE EKE 